\title{
A NOTE ON THE ERGODIC PROPERTIES OF HOMEOMORPHISMS
}

\author{
ROBERT SINE
}

\begin{abstract}
Powers of strongly ergodic transformations need not be strongly ergodic.
\end{abstract}

0 . Let $\phi$ be a homeomorphism of a compact Hausdorff space $X$. The asymptotic behavior of the iterates of $\phi$ has long been a subject of interest in ergodic theory. Aspects of the asymptotic behavior are best studied by means of the induced linear transformation on $C(X)$ defined by $T f(x)=f \circ \phi(x)$. This operator is easily shown to satisfy:

(1) $T 1=1$,

(2) $f \geqslant 0 \Rightarrow T f \geqslant 0$,

(3) $T(f g)=(T f)(T g)$

(4) $T$ is invertible.

We leave to the reader the easy task of showing that the properties characterize linear operators which are induced by homeomorphisms. A linear operator on $C(X)$ which satisfies only (1) and (2) is called a Markov operator. Much of what we investigate here is valid for such operators. Therefore certain of our definitions and results will be formulated in such a way that they make sense and are valid for Markov operators as well as the operators induced by homeomorphisms.

1. The system $(X, T)$ is called uniquely ergodic if there is exactly one invariant probability (normalized regular nonnegative Borel measure) $\mu$ for $T$. Oxtoby [7] has shown that in this case the Cesàro means

$$
A_{n}(T)=(1 / n)\left(I+T+T^{2}+\cdots+T^{n-1}\right)
$$

converge in the strong operator topology. (In this case necessarily to the one dimensional projection $\int f d_{\mu}$.) A unique invariant probability is not at all necessary for the strong operator convergence of the Cesàro means (for consider the identity homeomorphism). We will call $(X, T)$ strongly ergodic if the Cesàro means converge in the strong operator topology. The following result has been established for Markov operators in [8]. A contraction operator version was given in [9].

Presented to the Society, August 19, 1975; received by the editors December 10, 1974 and, in revised form, January 30, 1975.

AMS (MOS) subject classifications (1970). Primary 54H20, 34C35, 47A35, $60 \mathrm{~J} 05$.

Key words and phrases. Strongly ergodic transformations, Markov operator. 
THEOREM 1.T is strongly ergodic iff distinct invariant probabilities can be distinguished by invariant functions.

This is seen to contain the Oxtoby result. As an immediate corollary we obtain

Corollary. Suppose $T^{k}$ is strongly ergodic. Then $T$ itself is strongly ergodic.

Proof. Let $\mu_{1}$ and $\mu_{2}$ be distinct $T$-invariant probabilities. Clearly they are invariant for $T^{k}$ as well, so by Theorem 1 there is a function $f$ with $T^{k} f=f$ and $\left(f, \mu_{1}\right) \neq\left(f, \mu_{2}\right)$. Set $g=T f+\cdots+T^{k} f$ so that $T g=g$. Since $\left(T^{j} f\right.$, $\mu)=(f, \mu)$ for any $T$-invariant probability $\mu$, we have $\left(g, \mu_{1}\right) \neq\left(g, \mu_{2}\right)$. Thus by a second appeal to Theorem 1 we are done.

The purpose of this note is to demonstrate that the reverse implication of the Corollary is false. Before construction of the counterexample we will give some results which are valid in the reverse direction. First, more terminology. The system $(X, T)$ is strictly ergodic if it is uniquely ergodic and the support of the invariant probability is all of $X$. The following was proven in [5].

TheOREM 2. Let $(X, T)$ be strictly ergodic and $|\lambda|=1$. Then the operator $T_{\lambda}=(T / \lambda)$ is strongly ergodic iff $\lambda$ is not residual spectrum for $T$.

REMARK. If $\lambda$ is residual spectrum for $T$, then it is point spectrum for $T^{*}$. The $T^{*}$ eigenmeasure in this case is absolutely continuous with respect to the invariant measure. The Radon-Nikodym derivative is then a measurable eigenfunction for which there is no continuous version since $\lambda$ is not point spectrum. Certain theorems almost indicate that this cannot happen for homeomorphisms. A theorem of Kakutani (unpublished but see either Browder [1] or Hahn and Parry [4] for a proof) says if $(X, \phi)$ is minimal, $T f=\lambda f$ and $|f|=1$ (these last two equalities holding for all $x$ in $X$ ) and $f$ is a Baire function, then there is a continuous version. Furstenberg [3] has proven that with $(X, \phi)$ strictly ergodic if $T f=\lambda f$ [a.e., $\mu$ ] with $f$ in the first Baire class, then again there is a continuous version.

The next result indicates the connection between the existence of eigenfunctions and strong ergodicity of powers.

THEOREM 3. Suppose the operator $\left(T / \lambda^{r}\right)$ is strongly ergodic for $r=1$, $2, \ldots, m$ where $\lambda^{m}=1$. Then the operator $T^{m}$ is strongly ergodic.

Proof. Note that $\lambda+\lambda^{2}+\cdots+\lambda^{m}=0$. Compute for each value of $r=1,2, \ldots, m$ the sum

$$
S_{m n}\left(T / \lambda^{r}\right)=I+T / \lambda^{r}+\cdots+T^{m n} / \lambda^{r m n} .
$$

Now we sum these expressions from $r=1$ to $r=m$ and use the cancellation property of the roots of unity to obtain

$$
m S_{n}\left(T^{m}\right)=S_{m n}(T / \lambda)+\cdots+S_{m n}\left(T / \lambda^{m}\right) .
$$

It now follows at once that $A_{n}\left(T^{m}\right)$ converges so we are done.

REMARK. The theorem above implies that if $(X, T)$ is strictly ergodic and the $m$ th roots of unity do not belong to the residual spectrum of $T$ then $T^{m}$ is strongly ergodic. If $(X, \phi)$ is minimal then it is readily verified that either $(X$, $\left.\phi^{m}\right)$ is also minimal or $X$ decomposes into $X_{1} \oplus \cdots \oplus X_{k}$, a disjoint union 
of clopen subsets which are permuted by $\phi$ and $k$ divides $m$. Thus if $(X, \phi)$ is strictly ergodic and $\left(X, \phi^{m}\right)$ is not minimal then when $k=m$ the necessary continuous eigenfunctions can be written down directly as certain constants on each of the sets $X_{i}$ of the decomposition. In this case $\left(X, \phi^{m}\right)$ is strongly ergodic.

2. The algebraic point of view found in Ellis [2] suggests the following procedure for the construction of an example. Let $\beta Z$ denote the Stone-Čech compactification of the integers. The translation operator on $Z$ extends to a homeomorphism $\phi$ of $\beta Z$. Suppose that $\mathfrak{A}$ is a closed subalgebra of $C(\beta Z)$ which is invariant as a set under both $\phi$ and its inverse. Then $Y=\beta Z / \mathfrak{A}$ is a compact Hausdorff space obtained from $\beta Z$ by identification of points which cannot be distinguished by members of $\mathfrak{A}$. Since $\mathfrak{A}$ was stable as a set under the group generated by $\phi$ there is an induced homeomorphism $\hat{\phi}$ of $Y$. Let $\mu$ be any invariant probability for $(Y, \hat{\phi})$. Since $C(Y) \cong \mathfrak{A}$, we can regard $\mu$ as a norm 1 functional on the closed linear manifold $\mathfrak{A}$ in $C(\beta Z)$. Let $\mu_{1}$ be any Hahn-Banach extension of $\mu$ to all of $C(\beta Z)$. Then $\left\|\mu_{1}\right\|=\|\mu\|=1$ while (1, $\left.\mu_{1}\right)=(1, \mu)=1$. Thus $\mu_{1}$ is a probability on $\beta Z$. Now let $\mu_{2}$ be any cluster point of $\left\{A_{n}\left(T^{*}\right) \mu_{1}\right\}$. Then $\mu_{2}$ is an invariant probability for $(\beta Z, \phi)$ and is still an extension of $\mu$ since $\mu$ was invariant on $\mathfrak{A}$.

A probability $\lambda$ on $\beta Z$ is called a Banach limit if $\lambda$ is an invariant probability for the shift $\phi$. An almost convergent sequence $C=\left\{C_{n}\right\}$ is a member of $C(\beta Z)$ so that $(C, \lambda)$ is a constant independent of $\lambda$ for all $\lambda$ in $\Lambda$, the set of all Banach limits. In his paper setting down the entire foundations of the theory of almost convergent sequences, Lorentz [6] showed that the following condition is equivalent.

$(1 / n)\left(C_{1+r}+C_{2+r}+\cdots+C_{n+r}\right)$ converges to a constant independent of and uniformly in $r$ for $r$ in $Z$.

The set of all almost convergent sequences will be denoted by $\varrho$.

Suppose that the algebra $\mathfrak{A}$ of the first paragraph of this section satisfies $\mathfrak{U} \subset \mathcal{L}$. Then we claim that $(Y, \hat{\phi})$ is uniquely ergodic. We argue as follows. If $\mu_{1}$ and $\mu_{2}$ are distinct invariant probabilities on $Y$, then there is a function $f$ on $Y$ so that $\left(f, \mu_{1}\right) \neq\left(f, \mu_{2}\right)$. Now let $\sigma_{1}$ and $\sigma_{2}$ be any two Banach limits which are extensions of $\mu_{1}$ and $\mu_{2}$. Let $c$ in $\mathfrak{A}$ be the function on $\beta Z$ which is the lifting of $f$. Then $\left(c, \sigma_{1}\right) \neq\left(c, \sigma_{2}\right)$ contradicting the fact that $c$ is in $\mathcal{L}$. Thus when $\mathfrak{A}$ is contained in $\mathcal{L}$ we obtain a uniquely ergodic and, therefore, a strongly ergodic system as the quotient.

Let us make one final observation before the construction. If a system $(X$, $T$ ) is strongly ergodic, then for any $f$ and any $x$ the sequence of numbers $\left\{T^{n} f(x)\right\}$ is Cesàro limitable. Indeed, since $T$ is a contraction, the sequence is even almost convergent.

Now to construct the algebra. Let $c$ be a sequence constructed from small blocks of length two. Each small block is either $(1,-1)$ or $(-1,1)$. The small blocks are assembled into large blocks $\left\{\cdots B_{k}, B_{k+1}, \cdots\right\}$. Each $B_{k}$ is a string of two-blocks, all of the same kind, while $B_{k+1}$ will be a string of the other kind of two-block. We now specify the lengths of the $B_{k}$ blocks. If we take the subsequence of $c$ consisting of just the even terms, the subsequence will consist of strings of 1's alternating with strings of -1 's. If the $B_{k}$ block 
lengths are taken to grow sufficiently fast, the subsequence of even terms will fail to be Cesàro limitable. This is the only condition on block lengths we need to impose. This will guarantee that $\left(Y, \hat{\phi}^{2}\right)$ will not be strongly ergodic.

Now consider a monomial term $c_{1} c_{2} \cdots c_{n}$ where each $c_{i}$ is a translate of $c$. If we ignore a vicinity of the origin, the $B_{k}$ blocks of all of the $c_{i}$ 's almost overlap, and the two block phase in these translated $B_{k}$ blocks is the same as the phase of the two blocks in the translated $B_{k+1}$ blocks. Thus $c_{1} c_{2} \cdots c_{n}$ is seen to be in $\mathcal{L}$. Therefore polynomial terms with rational coefficients are also in $\mathcal{L}$. We take $\mathfrak{A}$ to be the closure of this algebra, and since $\mathcal{L}$ is closed we have $\mathfrak{A}$ in $\mathcal{L}$.

Thus $(Y, \hat{\phi})$ is uniquely ergodic and so is strongly ergodic while $\left(Y, \hat{\phi}^{2}\right)$ is not strongly ergodic. We conclude with mentioning that since $\mathfrak{A} \cong C(Y)$ is separable, the space $Y$ is compact metric.

\section{BIBLIOGRAPHY}

1. F. E. Browder, On the iteration of transformations in noncompact minimal dynamical systems, Proc. Amer. Math. Soc. 9 (1958), 773-780. MR 20 \#3456.

2. R. Ellis, Lectures on topological dynamics, Benjamin, New York, 1969. MR 42 \#2463.

3. H. Furstenberg, Strict ergodicity and transformations of the torus, Amer. J. Math. 83 (1961), 573-601. MR 24 \# A3263.

4. F. Hahn and W. Parry, Some characteristic properties of dynamical systems with quasi-discrete spectra, Math. Systems Theory 2 (1968), 179-190. MR 37 \#6435.

5. B Jamison and R. Sine, Irreducible almost periodic Markov operators, J. Math. Mech. 18 (1968/69), 1043-1057. MR 39 \#3589.

6. G. G. Lorentz, A contribution to the theory of divergent sequences, Acta Math. 80 (1948), 167-190. MR 10, 367.

7. J. C. Oxtoby, Ergodic sets, Bull. Amer. Math. Soc. 58 (1952), 116-136. MR 13, 850.

8. R. C. Sine, Geometric theory of a single Markov operator, Pacific J. Math. 27 (1968), 155-166. MR 39 \# 1630.

9. __ A mean ergodic theorem, Proc. Amer. Math. Soc. 24 (1970), 438-439. MR 40 \#5825.

Department of Mathematics, University of Rhode Island, Kingston, Rhode Island 02881 